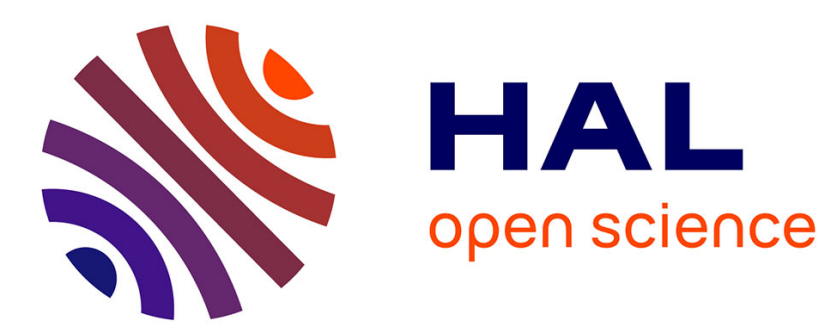

\title{
Cardiac and respiratory MRI gating using combined wavelet sub-band decomposition and adaptive filtering
}

Dima Abi-Abdallah, Agnès Drochon, Vincent Robin, Odette Fokapu

\section{To cite this version:}

Dima Abi-Abdallah, Agnès Drochon, Vincent Robin, Odette Fokapu. Cardiac and respiratory MRI gating using combined wavelet sub-band decomposition and adaptive filtering. Annals of Biomedical Engineering, 2007, 35 (5), pp.733-743. hal-01083996

\section{HAL Id: hal-01083996 https://hal.science/hal-01083996}

Submitted on 18 Nov 2014

HAL is a multi-disciplinary open access archive for the deposit and dissemination of scientific research documents, whether they are published or not. The documents may come from teaching and research institutions in France or abroad, or from public or private research centers.
L'archive ouverte pluridisciplinaire HAL, est destinée au dépôt et à la diffusion de documents scientifiques de niveau recherche, publiés ou non, émanant des établissements d'enseignement et de recherche français ou étrangers, des laboratoires publics ou privés. 


\title{
Cardiac and respiratory MRI gating using combined wavelet sub-band decomposition and adaptive filtering
}

\author{
Dima Abi-Abdallah ${ }^{1}$, Agnès Drochon $^{1}$, Vincent Robin $^{2}$, Odette Fokapu $^{1,3}$ \\ ${ }^{1}$ Laboratoire de Biomécanique et Génie Biomédical, Université de Technologie de Compiègne, France \\ ${ }^{2}$ Laboratoire de mathématique appliquées, Université de Technologie de Compiègne, France \\ ${ }^{3}$ Université de Picardie Jules-Verne, IUT de L’Aisne, France
}

Abbreviated title: MRI gating using wavelets and adaptive filtering

\section{Corresponding author:}

Dima Abi-Abdallah

Université de Technologie de Compiègne,

UMR CNRS 6600 Biomécanique et Génie Biomédical

BP20529 - 60205 Compiègne Cedex , France

Email:dima.abiabdallah@utc.fr

Phone: + 33344234423 / ext: 4750

Fax: + 33344237904 


\section{Abstract}

Cardiac Magnetic Resonance Imaging (MRI) requires synchronization to overcome motion related artifacts caused by the heart's contractions and the chest wall movements during respiration. Achieving good image quality necessitates combining cardiac and respiratory gating to produce, in real time, a trigger signal that sets off the consecutive image acquisitions. This guarantees that the data collection always starts at the same point of the cardiac cycle during the exhalation phase. In this paper, we present a real time algorithm for extracting a cardiacrespiratory trigger signal using only one, adequately placed, ECG sensor. First, an off-line calculation phase, based on wavelet decomposition, is run to compute an optimal QRS filter. This filter is used, afterwards, to accomplish $\mathrm{R}$ peak detection, while a low pass filtering process allows the retrieval of the respiration cycle. The algorithm's synchronization capabilities were assessed during mice cardiac MRI sessions employing three different imaging sequences, and three specific wavelet functions. The prominent image enhancement gave a good proof of correct triggering. QRS detection was almost flawless for all signals. As for the respiration cycle retrieval it was evaluated on contaminated simulated signals, which were artificially modulated to imitate respiration. The results were quite satisfactory.

\section{Keywords}

ECG, RLS, filter banks, real time synchronization. 


\section{Introduction}

The constantly evolving MR techniques have resulted in an increasing number of cardiac MRI scans in order to gain important insights into the functional and metabolic bases of heart disease. However, MRI observations of a moving organ, such as the heart, require synchronization so that the image acquisition might be accurately combined with its phase motion, thus eliminating the movement related blurring effects. It can be assumed that the heart moves independently with two motions: a contractile motion associated with the cardiac cycle, changing primarily the shape of the heart, and a respiratory motion that changes its position and orientation as the diaphragm and chest wall move with each breath [11]. Therefore, efficient synchronization techniques must associate cardiac gating with respiratory blanking, where the heart beat governed acquisitions are only enabled during at-rest periods (between breaths) to overcome the respiratory motion artifacts [4],[13],[15].

Cardiac gating is usually done by detecting the $\mathrm{R}$ peaks on the simultaneously recorded electrocardiogram (ECG), which are then used to trigger the consecutive image acquisition sequences. However, accomplishing an accurate $\mathrm{R}$ peak detection, hence a correct synchronization is often obstructed by the high corruption levels of the ECG signal due to electromagnetic effects. Especially when high resolution imaging is performed where high static magnetic fields as well as strong and fast switching gradients are needed. In fact the signal $\mathrm{S}(\mathrm{t})$, recorded in the NMR environment, is contaminated by many artifact signals :

- A signal due to patient related sensor motions in the magnetic field such as respiration, heart beat and voluntary motion. This artifact becomes more pronounced as the static magnetic field strength increases.

- A high frequency artifact resulting from the radio frequency pulses. This can be easily removed with low pass filtering. 
- Noise generated by the temporal variations of the magnetic field gradients. These are spurious signals that often resemble the QRS spikes and cause erroneous detection. In the case of small animal imaging, these artifacts are further aggravated by the exceptionally high heart rate (400-600 beats per minute), the high respiratory rate (30-60 breaths per minute) [4], and the low amplitude of the ECG signal (just a few millivolts).

- An artifact signal induced by the magnetohydrodynamic (MHD) effect. It results mainly in an elevation of the $\mathrm{T}$ wave that might reach amplitudes as high as the $\mathrm{R}$ wave, consequently leading to misgating.

Various approaches aiming to reduce the ECG signal artifacts have been previously considered in order to produce a synchronization signal. However, with the evolution of high resolution imaging techniques, the contamination levels have greatly increased, thus degrading the efficiency of classical analogical filtering methods [5],[7],[12]. Numerical methods were later proposed [2],[8], but could not be directly implemented in real time, yet it's indisputable that real time filtering is mandatory for achieving an efficient cardiac synchronization. Moreover the algorithm must account for the subject's physiological variations during the examination and for the artifact waveform variations, which differ according to the applied imaging sequence, as well as patient movements. Very few real time algorithms have been proposed [9] and [1], and the solutions impose various technical constraints. The first algorithm exploits a vectocardiogram (VCG) signal and necessitates the use of multiple sensors. However, positioning a large number of sensors in a NMR environment is not always simple, and might have consequences on the image quality. Not to mention the near impossibility of placing so many sensors on a small animal's chest. The second method, based on adaptive filtering, uses the machine's gradient signals as references. For this, one must have access to the MRI machine's gradient amplifier. In this paper, we propose a real time method, for achieving double cardiac and respiratory gating, that is quite simple and inexpensive in terms of materials. 
In order to combine cardiac gating with respiratory blanking, the respiration motion must be detected. This is usually done using motion detectors; however this is not always comfortable for some patients and is cumbersome for very small animals. Nevertheless, the respiration motion information is enclosed within the electrocardiogram as a modulating signal [6]: In fact, the respiratory motion of the thorax, heart and diaphragm changes the orientation of the heart's electric axis, resulting in an amplitude modulation of the ECG, which is itself a vectorial projection of the electrical activity of the heart.

In a previous work [2] we developed an off-line algorithm for cardiac triggering based on wavelet sub-band decomposition. By separating the sub-bands where the QRS energy is maximal, a reference signal was extracted from the ECG, and was then subjected to thresholding to produce a trigger signal. The algorithm has proven its efficiency even for very low signal to noise ratio (SNR) signals. In the present work we exploit this algorithm to produce an adapted filter, for real time use, that yields maximum QRS energy using the most appropriate wavelet. Furthermore, by applying an adequate electrode positioning, that maximizes the respiratory dependent modulation, we make use of the respiration information contained in the ECG signal in order to generate, in real time, a synchronization signal that takes into account the respiratory movements as well.

\section{Method}

\subsection{MRI technique and signal acquisition}

The MRI experimental setup (figure 1) incorporates a 2 Tesla OXFORD 85/310 horizontal cryomagnet with a $180 \mathrm{mT} . \mathrm{m}^{-1}$ gradient system using a home-made Alderman-Grant coil adapted to the small size of mice. A SMIS MR-operator control console, which has a scan management system with TTL signal input used as a timer for gated sequences. 
For cardiac and respiratory signal amplification and transmission outside the Faraday cage, an optical non-magnetic module was used. Signal detection was performed using 3M Red Dot neonatal electrodes, (3M, St Paul, MN). The ECG active electrodes were placed parallel to the heart axis $\left(45^{\circ}\right)$ in order to obtain maximum modulation. The respiration modulated ECG signals were thus recorded during MR scanning according to three high resolution imaging sequences - Gradient Echo (GE), Fast Spin Echo (FSE) and Inversion Recovery Spin Echo (IRSE)- inducing different types of contamination signals. For all three sequences, coronal and transversal planes were imaged. 4 to 6 slices of $1 \mathrm{~mm}$ thickness were acquired with an in-plane resolution of $100 \mu \mathrm{m}$, a field of view (FOV) of $27 \mathrm{~mm}$ and a flip angle $(\alpha)$ of $60^{\circ}$. MR images were reconstructed using a matrix of 256 x 256 pixels. Repetition time (TR) to echo time (TE) ratios were 385/10 ms for GE, and 2240/50 ms for T2-weighted FSE and IRSE. The system for digital processing of the contaminated modulated ECG, used for sampling, filtering and gating signal generation, consists of a National Instruments' DAQCard 6024E and a real time structure developed using Matlab ${ }^{\circledR}$ \& Simulink $^{\circledR}$ (The MathWorks Inc., Natick, MA).

\subsection{Cardiac-respiratory trigger extraction process}

The cardiac-respiratory trigger extraction process, illustrated in figure 2 , is made up of two simultaneous trigger production operations: the R peaks of the ECG are detected to yield a cardiac trigger, while the respiratory modulation signal is extracted, from that same ECG, to produce the respiration trigger. The final synchronization signal is none other than the combination of both triggers (logical AND) so that the image acquisitions would only be set off by the $\mathrm{R}$ waves during the low respiratory phase.

\subsubsection{Theoretical Basis}




\section{i. QRS detection}

ECG signals are characterized by a cyclic occurrence of patterns (QRS complexes, $\mathrm{P}$ and T waves) with different frequency contents. Power spectral analysis of the ECG show that, $\mathrm{P}$ and $\mathrm{T}$ waves only present a significant spectral density up to $10 \mathrm{~Hz}$, and that most of the QRS power lies in the 3-20 Hz band, beyond which the complex energy decreases gradually [14]. Thus, a strategical approach for detecting heartbeats would be to analyze different sub-bands of the ECG using wavelet based filter banks [3]. Figure 3 shows an illustration of a previously developed algorithm [2]. It consists in decomposing the ECG signal into multiple scales ( 8 for a signal sampled at $f_{s}=1 \mathrm{Khz}$ ), then reconstructing the details and keeping only those that contain the maximum of the QRS energy in order to compose a reference signal. This signal is then subjected to a double threshold comparator to produce a cardiac trigger. The algorithm was tested on simulated as well as small rodents ECGs that had been contaminated by one of three imaging sequences (Gradient Echo, Fast Spin Echo and Inversion Recovery Spin Echo) and some analyzing wavelets revealed having outstanding performances according to the signal and the sequence type.

But even though the wavelet decomposition algorithm gave proof of an almost infallible QRS detection, it could not be applied in real time for image synchronization, because the decomposition into 8 levels followed by the reconstruction of the $6^{\text {th }}$ and $7^{\text {th }}$ detail induce a significant delay. Equation (1) gives the time necessary for the reconstruction of the $\mathrm{n}^{\text {th }}$ detail using a wavelet with $\mathrm{M}$ coefficients

$$
\frac{1}{f}\left(2^{n}-1\right)(M-1)
$$


For instance, if we use the Db6 wavelet $(\mathrm{M}=6)$, to reconstruct the 7 th detail of a signal $(n=7)$ sampled at $f_{s}=1000 \mathrm{~Hz}$ we get a 0,635 seconds delay, which is almost equal to one heart cycle.

In order to achieve real time synchronization, while making use of the wavelets capabilities, we conceived an off-line pre-calculation phase, that precedes the actual real time trigger generation. It consists of a coefficients computation phase, where an optimal QRS filter is calculated based on wavelet decomposition, which can afterwards be easily used for real time processing.

The purpose of this first stage is to construct a filter that would yield the same reference signal as the wavelet decomposition method while assuring a minimum delay. A good approach for this filter optimization problem is to minimize the mean square value of the error signal that is defined as the difference between the desired response and the actual filter output. For stationary inputs, the resulting solution is commonly known as the Wiener filter, which is said to be optimum in the mean square sense. However the Wiener filter is inadequate for dealing with situations in which the signal and/or the noise are non stationary (such as the ECG). In such situations the filter has to assume a time varying form. An efficient method for realizing this is to use an adaptive filter.

\section{Adaptive filter}

By such a device we mean one that is self designing, in that the adaptive filter relies for its operation on a recursive algorithm which makes it possible for the filter to perform satisfactorily in an environment where complete knowledge of the relevant signal characteristics is not available. In a non stationary environment the algorithm offers a tracking capability whereby it can track time variations in the statistics of the input data provided that the variations are sufficiently slow. As a discrete consequence of the application of a recursive algorithm, where the parameters of an adaptive filter are 
updated from one iteration to the next, the parameters become data dependent. The operation of an adaptive filtering algorithm involves two basic processes: a filtering process designed to produce an output in response to a sequence of input data, and an adaptive process, the purpose of which is to provide a mechanism for the adaptive control of an adjustable set of parameters used in the filtering process. These 2 processes work interactively with each other.

There is no unique approach to the development of an adaptive filter; there is a variety of recursive algorithms each of which offers desirable features of its own. The most popular solutions are the LMS (least mean square) and the RLS (recursive least square). In this study we preferred the RLS approach because it is more robust than the LMS, and it has a faster convergence rate: Contrarily to the LMS, where the correction, applied in updating the old estimate of the coefficient vector, is based on the instantaneous sample value of the tap-input vector and the error signal, the RLS utilizes all the information contained in the input data from the start of the adaptation up to the present [10]. Since it converges fast, the RLS filter computation allows us to record a smaller chunk of signal to be used in this stage. The superior performance of the RLS algorithm compared to the LMS, however, is attained at the expense of a large increase in computational complexity. But this is of no inconvenience to us since we operate in an off-line computation process, and we only have a small number of coefficients (short filter delay) to calculate.

\section{Summary of the RLS algorithm:}

1. $\quad$ let $n=1$

2. compute the gain factor: $\mathbf{k}(n)=\frac{\mathbf{P}(n-1) \mathbf{u}(n)}{1+\mathbf{u}^{T}(n) \mathbf{P}(n-1) \mathbf{u}(n)}$

3. compute the true estimation error : $\eta(n)=d(n)-\mathbf{u}^{T}(n) \hat{\mathbf{h}}(n-1)$

4. $\quad$ update the estimate of the coefficient vector: $\quad \hat{\mathbf{h}}(n)=\hat{\mathbf{h}}(n-1)+\mathbf{k}(n) \eta(n)$

5. $\quad$ update the error correlation matrix: $\mathbf{P}(n)=\mathbf{P}(n-1)-\mathbf{k}(n) \mathbf{u}^{T}(n) \mathbf{P}(n-1)$ 
6. increment $n$ by 1 and go to step 2

where

$n:$ current iteration number

$u(n)$ : input at iteration $n$

$P(n)$ :inverse of the correlation matrix

$k(n)$ : gain vector

$\hat{h}(n):$ filter coefficient vector

$\eta(n)$ : estimation error

$d(n):$ desired output

\section{i. Respiratory phases delineation}

It is well-known that respiration influences electrocardiographic measurements. During the respiratory cycle, chest movements and alterations in thoracic impedance, as the lungs fill and empty, alter the ECG morphology. In fact, the ECG is a projection of the heart's electrical activity vector along the derivation direction (as perceived by the leads). The respiratory motion of the thorax, heart and diaphragm changes this vector's orientation, resulting in an amplitude modulation of the ECG in the direction of the cardiac mean electrical axis. It has been shown [6] that positioning the ECG electrodes along the heart axis $\left(45^{\circ}, \mathrm{V} 2 \mathrm{~V} 3\right)$ can maximize the respiratory dependent modulation, thus facilitating the extraction of the respiratory information without however affecting the cardiac signal quality. Note that when electrodes are placed at this position, inhaling would produce a decrease of amplitude (figure 4).

An easy way to retrieve the respiratory modulating signal is to apply a highly selective low pass filter L(n) to the recorded ECG. Using Matlab ${ }^{\circledR}$ 's Digital Filter Design Tool we calculated a FIR equiripple filter. Note that the equiripple design produces the most 
efficient filters, that is, that they just meet the specification with the least number of coefficients. A cut-off frequency of $1.2 \mathrm{~Hz}$ was deemed acceptable since the rodents respiration frequency is usually less than $1 \mathrm{~Hz}$, especially when anesthetized, as is the case during NMR imaging. Naturally, it comes to one's attention that due to the narrow bandwidth of the low pass filter, the extracted respiratory signal would be greatly delayed. In fact, the designed filter L(n) has over 3000 coefficients (for a sampling frequency of $0.5 \mathrm{Khz}$ ) leading to an approximate 1.5 seconds delay, thus compromising the respiratory synchronization. Nonetheless, given that the respiration is somewhat periodic, the delay can be simply compensated by a prediction of the upcoming signal along a period which value is continuously updated.

\subsubsection{Algorithm}

\section{i. Off- line filter computation phase}

The off-line phase algorithm proceeds as follows:

- Record a small portion of signal $\mathrm{S}_{\text {ori }}(\mathrm{t})$ (minimum duration of two or three respiratory periods ) while the imaging sequence gradients are on.

- Process it to produce a reference signal $S_{\text {ref }}(t)$ based on the previous method [2] using the most appropriate wavelet.

- Calculate the optimal filter coefficients using the RLS algorithm by setting $\mathrm{S}_{\text {ori }}(\mathrm{t})$ as input and $\mathrm{S}_{\mathrm{ref}}(\mathrm{t})$ as the desired signal. A number of 64 coefficients for the filter seemed to provide a good compromise for getting low error while having short delay and computation time. (the larger the coefficients number, the smallest the error but with longer delay and computation time)

Thus we obtain a filter $\mathrm{F}(\mathrm{n})$ that is adapted to both the ECG signal and the applied imaging sequence. 


\section{ii. $\quad$ Real time trigger production}

This second phase consists of two simultaneous processes: an $\mathrm{R}$ peak detection producing a cardiac trigger, along with a respiratory trigger extraction. The final cardiac-respiratory signal used for triggering the imaging sequences is composed by combining both triggers.

\section{Cardiac trigger}

The recorded NMR contaminated signal is filtered by the calculated minimum delay $\mathrm{F}(\mathrm{n})$ to obtain an artifact free ECG, where the QRS peaks constitute the most prominent features. This signal can then undergo a simple threshold comparison to produce the cardiac trigger. Note that the threshold values are adjustable via a graphical interface.

\section{Respiratory trigger}

The collected ECG is filtered using the selective low pass filter $\mathrm{L}(\mathrm{n})$, to produce the respiration related modulating signal. Then, the respiratory gating trigger is produced by subjecting the filtered signal to a simple threshold at the mean value.

The algorithm described above was implemented on Simulink ${ }^{\circledR,}$ s Real Time Windows Target platform. The conceived Simulink ${ }^{\circledR}$ model (outlined in figure 5) was compiled using Matlab ${ }^{\circledR}$ s Real Time Workshop, to create a real time software application. The application was then attached to a user friendly interface (figure 6) that allows the experimenter to launch the different procedures, and to easily pick and set the algorithm's parameters: At the beginning of the session, after recording a chunk of signal, he can pick a wavelet, test its performances and adjust the $\mathrm{R}$ peaks detection thresholds. Once the wavelet is selected, he can run the off-line calculation phase, and then start the online trigger production. 


\section{Results}

The cardiac-respiratory trigger extraction algorithm was tested for mice cardiac MRI synchronization, using three imaging sequences GE, FSE and IRSE. The animal study was conducted with approval from the Institutional Animal Care and Use Committee of the University of Lyon 1 . The mice were anesthetized by intraperitoneal injections of pentobarbital sodium (50 mg/kg, Sanofi, France).

According to the applied sequence the algorithm was run using the most appropriate wavelet [2]: Coif3 for GE, Sym8 for FSE and Sym4 for IRSE. The real time process was able to generate effective online cardiac-respiratory triggering for all 3 sequences. An illustration is given in figure 7. The QRS peak detections, achieved in real time by the adapted filter, were later compared to the triggers that the wavelets would have produced off-line using the prior algorithm. For each sequence, the detection error rates were as low as those given by the best performing wavelets [2]. Furthermore, we did not perceive any significant influence of slice orientation on the gating process. The error rates were similar whether the signals were acquired during coronal or transversal slice acquisition. In all cases we attained an almost perfect QRS detection.

The evaluation of the respiration detection algorithm was carried out on simulated signals. In fact, it was difficult to delineate the respiration phases manually because the sequence noise alters the $\mathrm{R}$ peak amplitude thus preventing correct visual detection of the signal modulation. Previously recorded contaminated ECGs [2] were modulated by a sine wave that comprises variations of amplitude (up to $150 \%)$ and frequency $(0.2 \mathrm{~Hz}-1 \mathrm{~Hz})$. The algorithm performed just as expected: the modulation was extracted successfully, however when a change in frequency and/or amplitude occurs the algorithm takes around one respiration period or two to adapt to the changes. During that time, a high respiration phase might be mistaken for a low one and taken 
into account for synchronization. Once the algorithm recovers, it will once again correctly retrieve the modulating signal (figure 8). Note that the modulation retrieval was independent of the sequence type; we observed the same behavior with all three sequences (the noise is eliminated by the low pass filter).

The global evaluation of the algorithm was done in terms of image quality. The images, acquired with synchronization, revealed a very clear enhancement compared to cardiac MRIs taken without cardiac or respiratory triggering (figures 9, 10 and 11). Without any synchronization the image is quite blurry especially in the parts that move along with each heart beat. With cardiac triggering, clearer structures appear on the image close to the heart. When applying both cardiac and respiratory synchronization, we observe a better contrast in the whole image, and the structures are more emphasized.

\section{Discussion}

Cardiac-respiratory synchronization is essential for obtaining good quality cardiac MR images. However, the perturbation of the ECG signals acquired in the NMR environment, especially during small animal imaging, greatly obstructs gating tasks.

In this work, a method for triggering MR imaging sequences on cardiac and respiration rate is presented. An adapted filtering, based on wavelet sub-band decomposition, is applied to the contaminated simultaneously recorded modulated ECG to rid it of the NMR environment artifacts, while emphasizing the QRS peaks. This allows the extraction of a cardiac trigger that is completely synchronized with the heart beats. The presence of an off-line pre-calculation phase is very convenient, in the sense that we can add any kind of operation to the wavelet process to improve the reference signal, and then regroup all of it in a single filter with minimum delay.

In addition, based on the fact that the amplitude of the ECG varies according to the respiration motions, a demodulation of this "cardiac respiratory" signal yields a respiration curve which is 
used to build the respiratory triggering signal. A final trigger is then obtained by combining both cardiac and respiratory triggers and used for MRI gating. The algorithm has proven efficiency for small rodents' cardiac imaging. The trigger extraction was almost flawless and the image quality was greatly enhanced.

Our method could constitute a good alternative for the solutions proposed by Fischer et. al. [9] and Abächerli et. al. [1]. Unlike these solutions, the technique presented in this paper does not have any technical constraints. The use of one ECG sensor is quite practical and can be easily positioned even for small animals. Furthermore, the extracted reference signal is independent of the MRI machine.

As for the algorithm, the following remarks can be discussed:

Adaptive improvement: For images which acquisitions take a relatively long time to complete, significant variations may occur in the signal morphology. Therefore, better results would be obtained if the filter coefficients were recalculated during the synchronization operation and updated every few minutes, in order to adapt the processing to the signal changes. Nevertheless, since no major signal variations occurred in all ECGs recorded throughout our experiments, using the same filter during the whole imaging session was just as good as using updated filters. That is why calculating the filter only in an off-line pre-processing phase was satisfactory enough. However, in a future work we will incorporate continuous filter estimations and coefficients updating during the real time process. We will also give the user the possibility to adjust the $\mathrm{R}$ peak detection thresholds during the online phase.

Off-line phase duration: The length of the signal to be used for the pre-calculation phase can be defined by the experimenter as he sees adequate. However a minimum duration of two or three respiratory periods is required so that the algorithm could function properly. Even though the RLS convergence is a lot faster than that, additional time is needed to make an initial estimation of some parameters that are necessary to get the respiration trigger production started. 
Respiration extraction: the implemented respiration extraction algorithm could be evaluated more effectively by using a traditional respiration detector, and comparing the extracted signal with that of the detector. However placing any additional detector on the mice was nearly impossible given the small thorax area. We could also use a ventilator system and impose breathing frequency changes; nevertheless, given the narrow dimensions of the MRI tunnel and of the antenna, which have been maximally reduced to enhance the image quality, placing such system would be very difficult. It is conceivable, though, to test it on human subjects in a later stage. For the time being we have only evaluated the algorithm using simulated signals. The modulation variations imposed on these signals imitated, as close as possible, the variations that would most likely occur in real signals: smooth frequency and amplitude modifications. In that case, errors would only occur during the respiration period that follows the change. Even so, this did not seem to impair the image enhancement. Note that in order for the method to function properly, there has to be steady stages where the signal is not altered. For example, if the signal has a frequency that is constantly changing, say in a steep slope linear manner, the detection error rate suffers a great deal. In fact, the algorithm needs to predict the upcoming period, so if the latter keeps changing the prediction would always be faulty.

The effect of cardiac pathologies on the performances of the extraction method still needs to be examined more thoroughly. It has come to our attention that some diseases, might introduce very low frequency components into the ECG signal. These components could get through the low pass filter, and distort the extracted modulating signal. The filtered signal would not only contain the respiration but it will also include some additional disruptions.

\section{Conclusion}

The method proposed in this paper for synchronizing image sequences on the subject's physiological rhythm is simple and original. Using one ECG sensor to achieve double cardiac 
and respiratory synchronization is a potentially useful technique, especially in the NMR environment where the number of measurement devices must be as limited as possible. The efficiency of the method relies mainly on the idea of combining the wavelet transform with adaptive filtering, thus solving, with this association, most frequently encountered difficulties in such applications. The developed user interface allows the experimenter to optimize the denoising parameters according to the MRI sequence type, and enhance the trigger signal quality throughout the imaging session. The algorithm has been successfully applied to modulated ECG signals recorded on healthy small animals. It now needs to be tested on signals recorded in pathological cases. 


\section{Acknowledgments}

The study was supported by the Ministry of Industry of France (RNTS 2003).

The authors would like to thank the "Laboratoire de RMN, UMR CNRS 5012",University Claude Bernard Lyon 1 for the use of their MR machine and the support by their MR staff. Another thank you is addressed to the J. Felblinger of the IADI laboratory University Hospital Nancy,Brabois at Vandoeuvre-les-Nancy for his technical help. 


\section{References}

1. Abächerli, R., C. Pasquier, F. Odille, M. Kraemer, J.J. Schmid and J. Felblinger. Suppression of MR gradient artefacts on electrophysiological signals based on an adaptive real-time filter with LMS coefficient updates. MAGMA. 18:41-50, 2005.

2. Abi Abdallah, D., E. Chauvet, L. Bouchet-Fakri, A. Bataillard, A. Briguet and O. Fokapu. Reference signal extraction from corrupted ECG using wavelet decomposition for MRI sequence triggering: application to small animals. Biomed. Eng. Online. 5:11, 2006.

3. Afonso, V.X., W.J. Tompkins, T.Q. Nguyen, and S. Luo. ECG Beat Detection Using Filter Banks. IEEE Trans. Biomed. Eng. 46(2):192-202, 1999.

4. Cassidy, P.J., J.E. Schneider, S.M. Grieve, C. Lygate, S. Neubauer, and K. Clarke. Assessment of Motion Gated Strategies for Mouse Magnetic Resonance at High Magnetic Fields. J. Magn. Reson. Imag. 19: 229-237, 2004.

5. Damji, A.A. and R.E. Snyder. Interference suppression in cardiac synchronization system operating in a high magnetic field NMR imaging system. Magn. Reson. Med. 6:89-95, 1988.

6. Felblinger, J., and C. Boesch. Amplitude Demodulation of the Electrocardiogram Signal for Respiration Monitoring and Compensation during MR Examinations. Magn. Reson. Med. 38: 129-136, 1997.

7. Felblinger, J., C. Lehmann, and C. Boesch. Electrocardiogram Acquisition during MR Examinations for Patient Monitoring and Sequence Triggering. Magn. Reson. Med. 32:523-529, 1994.

8. Felblinger, J., J. Slotboom, R. Kreis, B. Jung, and C. Boesch. Restoration of Electrophysiological Signals Distorted by Inductive Effects of Magnetic Field Gradients during MR Sequences. Magn. Reson. Med. 41 : 715-721, 1999. 
9. Fischer, S.E., S.A. Wickline, and C.H. Lorenz. Novel real-time R-wave detection algorithm based on the vectorcardiogram for accurate gated magnetic resonance acquisitions. Magn. Reson. Med. 42(2):361-370, 1999.

10. Haykin, S. Standard recursive least-squares estimation in adaptive filter theory. Prentice Hall International Editions. 1991, pp. 477--507.

11. Klein, G.J., B.W. Reutter, M.H. Ho, J.H. Reed, and R.H. Huesman. Real-time system for respiratory-cardiac gating in positron tomography. IEEE Trans. Nucl. Sci. 45: 21392143, 1997.

12. Laudon, M.K., J.G. Webster, R. Frayne, and T.M. Grist. Minimizing Interference from Magnetic Resonance Imagers During Electrocardiography. IEEE Trans. Biomed. Eng. 45(2): 160-164, 1998.

13. Ma1, W., C.T. Badea, C.T. Wheeler, L.W. Hedlund, and G.A. Johnson. Effects of Breathing and Cardiac Motion on Spatial Resolution in the Microscopic Imaging of Rodents. Magn. Reson. Med. 53:858-865, 2005.

14. Thakor, N.V., J.G. Webster, and W.J. Tompkins. Estimation of QRS Complex Power Spectra for Design of a QRS Filter. IEEE Trans. Biom. Eng. 31(11):702-706, 1984.

15. Wiesmann F., M. Szimtenings, A. Frydrychowicz, R. Illinger, A. Hunecke, E. Rommel, S. Neubauer, and A. Haase. High-resolution MRI with cardiac and respiratory gating allows for accurate in vivo atherosclerotic plaque visualization in the murine aortic arch. Magn. Reson. Med. 50:69-74, 2003. 


\section{Figure Legends}

\section{Figure 1 - MRI gating experimental setup}

Signal acquisition is achieved using a MR compatible ECG sensor. The signals are optically transmitted outside the magnet and then fed into the processing module which generates a synchronization signal that will command the image sequence triggering.

\section{Figure 2 - Real time cardiac-respiratory trigger extraction:}

The signal is subjected to an adapted filtering to enable QRS detection, as well as a low pass filtering to extract the respiration signal. The cardiac and respiratory triggers are then combined to produce a synchronization signal that controls the image sequence triggering

\section{Figure 3 - Overview of the off-line cardiac trigger extraction algorithm}

The contaminated ECG is decomposed into 8 scales; the reference signal is then constructed by adding together the $6^{\text {th }}$ and $7^{\text {th }}$ sub-band signals. A simple trigger generates the cardiac rhythm as well as the synchronization signal.

Hi-D and Hi-R are respectively the high pass decomposition filter and its associated reconstruction filter. Lo-D and Lo-R represent the low pass decomposition and reconstruction filters. $\mathrm{cD}_{\mathrm{j}}$ denotes the detail coefficients, and $\mathrm{d}_{\mathrm{j}}$ are the reconstructed detail signals.

\section{Figure 4 - Respiratory modulation of the ECG signal}

The respiratory dependent modulation of the ECG signal is maximized by placing the electrodes along the heart axis. The highest $\mathrm{R}$ amplitude is reached during the expiration phase.

\section{Figure 5 - Simulink® bloc model}

Outline of the algorithm's bloc implementation under Simulink ${ }^{\circledR}$. The analog input bloc acquires the data from the NI DAQCard and feeds it into 2 simultaneous processing blocs. The first one 
applies the adapted filter and detects the $\mathrm{R}$ peaks. The second one retrieves the respiration signal by low pass filtering and delay compensation. Then the respiratory trigger is produced by thresholding the respiration signal to a dynamically adapting mean value. Having both cardiac and respiration triggers the final trigger is produced and sent to the acquisition card's output channel.

\section{Figure 6 - The synchronization software's graphical user interface}

It allows the user to execute different tasks, such as testing the wavelets and threshold values to choose the optimal settings, defining the running times, and commanding the program execution.

\section{Figure 7 - Synchronization signal extraction:}

Cardiac-respiratory trigger extraction from a mouse ECG recorded during the FSE sequence. The final trigger reproduces the occurrences of the $\mathrm{R}$ peaks only during the exhalation phase i.e. when the respiratory modulation signal is higher than its mean value.

\section{Figure 8 - Trigger extraction from a simulated ECG:}

Simulated ECG signal acquired during the GE sequence, and modulated by a varying sine wave. Around the $28^{\text {th }}$ second, the wave's frequency is doubled and its amplitude is reduced by $40 \%$. During the adaptation period the algorithm misses 3 peaks and detects 2 false ones. Otherwise all detected peaks are correctly detected within the low respiration phase. Note that the represented modulating signal is shifted for visibility's sake.

\section{Figure 9 - Mouse cardiac MRIs acquired with a GE sequence:}

Gating during coronal multislice GE: (a) with no synchronization, (b) with cardiac gating, (c) with cardiac-respiratory gating. Gated compared to non-gated images show more details and fewer motion artifacts. 
Figure 10 - Mouse cardiac MRIs acquired with a FSE sequence:

Gating during transversal multislice FSE: (a) with no synchronization, (b) with cardiac gating, (c) with cardiac-respiratory gating. The aortic arch details are clearly visualized with cardiacrespiratory gating.

Figure 11 - Mouse cardiac MRIs acquired with an IRSE sequence:

Gating during coronal multislice IRSE: (a) with no synchronization, (b) with cardiac-respiratory gating. 